\title{
Circulating MicroRNAs as Novel Potential Biomarkers for Left Ventricular Remodeling in Postinfarction Heart Failure
}

\author{
Guangyuan Gao $\mathbb{D}^{1,2}$ Weiwei Chen, ${ }^{1,2}$ Miao Liu, ${ }^{1,2}$ Xu Yan, ${ }^{1,2}$ and Ping Yang $\mathbb{D}^{1,2}$ \\ ${ }^{1}$ Department of Cardiology, China-Japan Union Hospital of Jilin University, Changchun 130031, China \\ ${ }^{2}$ Jilin Provincial Molecular Biology Research Center for Precision Medicine of Major Cardiovascular Disease, \\ Changchun 130031, China
}

Correspondence should be addressed to Ping Yang; pyang@jlu.edu.cn

Received 18 June 2019; Revised 10 September 2019; Accepted 15 November 2019; Published 2 December 2019

Guest Editor: Alexander Berezin

Copyright ( 92019 Guangyuan Gao et al. This is an open access article distributed under the Creative Commons Attribution License, which permits unrestricted use, distribution, and reproduction in any medium, provided the original work is properly cited.

Circulating microRNAs (miRNAs) have been proposed as potential biomarkers for left ventricular remodeling in postinfarction heart failure (HF). However, the diagnostic reproducibility of the use of circulating miRNAs may be affected by the temporal expression of miRNAs following myocardial infarction (MI). In the current study, using a MI-induced HF rat cohort (4-, 8-, and 12-week post-MI groups), we investigated the temporal expression of plasma miRNAs during the development of left ventricular remodeling. The plasma miRNA expression profile was obtained using miRNA sequencing. The expression of candidate miRNAs in plasma and tissues was examined with real-time PCR. Target genes of candidate miRNAs were predicted using a parallel miRNA-messenger RNA expression profiling approach. The value of plasma miRNAs as biomarkers for left ventricular remodeling was evaluated in patients with postinfarction $\mathrm{HF}(n=32)$ and control patients with stable angina and without significant coronary lesions and HF $(n=16)$ with real-time PCR. Although the expression levels of miR-20a-5p, miR$340-5 p$, and let-7i-5p were temporally regulated in plasma, myocardium, and peripheral blood mononuclear cells, the expression levels of plasma miRNAs, especially miR-20a-5p, were associated with the development of left ventricular remodeling in the postinfarction HF rat cohort. The target genes of these 3 miRNAs were associated with the mechanistic target of rapamycin, nuclear factor- $\kappa \mathrm{B}$, tumour necrosis factor, apoptosis, and p53 signaling pathways. Additionally, the plasma levels of miR-20a-5p, miR-340-5p, and let-7i-5p were significantly increased in patients with postinfarction HF. However, only the expression levels of miR-20a-5p presented significant positive correlations with left ventricular internal end diastolic dimension and left ventricular end diastolic volume. In conclusion, the expression levels of plasma miR-20a-5p were significantly associated with the degree of left ventricular dilatation, and plasma miR-20a-5p may be a potential biomarker for postinfarction left ventricular remodeling.

\section{Introduction}

Heart failure (HF) is one of the leading causes of hospital admission and mortality worldwide [1]. Myocardial infarction (MI) is a common predisposing cause of HF [1]. Following MI, progressive left ventricular remodeling occurs in the noninfarcted myocardium and plays a central role in the pathophysiology of HF $[2,3]$. Left ventricular remodeling is characterized by left ventricular dilatation, hypertrophy, distortion of contour, activation of interstitial fibrosis, and deterioration in cardiac dysfunction [2-4]. In clinical practice, circulating biomarkers such as natriuretic peptides associated with cardiac overload facilitate the clinical management of
HF [1]. Nevertheless, there is a need to find reliable circulating biomarkers for tracking the process of left ventricular remodeling to help clinicians recognize high-risk patients for more aggressive prevention as early as possible.

Recently, circulating microRNAs (miRNAs) have become an emerging class of biomarkers. miRNAs are a large class of small noncoding RNAs that are powerful posttranscriptional regulators of gene expression [5]. Gene expression reprogramming is the base of ventricular remodeling [6]. Several studies have demonstrated that miRNAs are involved in the development of HF by silencing the expression of genes [79]. As miRNAs can be packaged in microparticles (exosomes, microvesicles, and apoptotic bodies) or bound to transport 
proteins, they are protected from degradation and can be detected by quantitative methods in the plasma samples [10]. Recent clinical studies have indicated that circulating miRNA may be valuable biomarkers for postinfarction left ventricular remodeling [11-18]. However, the diagnostic reproducibility of using circulating miRNAs as biomarkers for postinfarction cardiac remodeling remains imperfect, which may be due to their time-dependent expression during the development of $\mathrm{HF}$, the complex mechanisms underlying the progression of cardiac remodeling, and the use of antiremodeling medical therapy [11-18].

The rat coronary artery ligation model exhibits many pathophysiologic and clinical characteristics that are similar to MI-induced HF in humans [19]. Progressive left ventricular dilatation can be continuously observed for up to 3-4 months following coronary artery ligation [19]. In the present study, using a rat cohort (4-, 8-, and 12-week postcoronary artery ligation groups), we assessed the temporal expression of plasma miRNAs during the development of left ventricular remodeling and conducted a preliminary study to explore the possible origins and target genes of candidate miRNAs. The value of candidate plasma miRNAs as biomarkers for postinfarction left ventricular remodeling was subsequently examined in a case control study between patients with postinfarction $\mathrm{HF}$ and patients with stable angina (without significant coronary lesions and HF).

\section{Materials and Methods}

2.1. Animals. All animal procedures were conducted in accordance with the institutional guidelines for the care and use of laboratory animals at Jilin University, Jilin, China. All experimental procedures were approved by the Ethical Review Board of China-Japan Union Hospital of Jilin University. Male Wistar rats ( 8 weeks, weighing 250 280 g) were obtained from the Center for Laboratory Animals, Medical College, Jilin University, Changchun, China. The animals were subjected to sham surgery or surgery involving ligation of the left anterior descending artery. Briefly, the rats were anaesthetized with oxygen containing $3 \%$ isoflurane supplied by a rodent respirator. Following anaesthetization, the thorax was opened in the left parasternal area, and MI was induced by ligating the left anterior descending coronary artery using 3-0 suture between the pulmonary cone and left atrium. After 4 weeks, successful induction of HF was confirmed by echocardiography, and the animals were randomly divided into 4 -week $(n=16), 8$-week $(n=20)$, and 12 -week $(n=16)$ postMI groups and corresponding sham groups $(n=12, n=16$, and $n=12$ ). At 4 -week, 8 -week, and 12 -week post-MI induction, echocardiography was recorded. Animals were then euthanized, and the samples (heart, liver, spleen, lung, kidney, thymus, and blood) were quickly harvested. Tissue samples were conserved in liquid nitrogen until use. Blood was collected in ethylenediaminetetraacetic acid-treated tubes. Following preliminary centrifugation $\left(2,000 \times \mathrm{g}, 10\right.$ minutes, $\left.4^{\circ} \mathrm{C}\right)$, the plasma samples were further centrifuged $(16,000 \times \mathrm{g}, 10$ minutes, $4^{\circ} \mathrm{C}$ ) to pellet platelets and cellular debris, and the aliquots of supernatant were stored at $-80^{\circ} \mathrm{C}$ in a freezer.
Peripheral blood mononuclear cells (PBMCs) were purified with Ficoll separating solution (TBDscience, Tianjin, China).

2.2. Patient Population. Patients with postinfarction $\mathrm{HF}$ admitted to China-Japan Union Hospital of Jilin University between December 2018 and June 2019 were eligible for the current study. Prior MI and HF were defined according to the current definitions $[1,20,21]$. Patients who met the criteria "pathological Q waves, in the absence of nonischemic causes and/or imaging evidence of the loss of viable myocardium in a pattern consistent with ischemic etiology" were diagnosed as prior MI [20]. Patients with symptoms and/or signs of HF, N-terminal pro-B type natriuretic peptide $(\mathrm{NT}-\operatorname{proBNP})>125 \mathrm{pg} / \mathrm{ml}$, and other cardiac functional and structural alterations underlying $\mathrm{HF}$ were diagnosed as HF $[1,21]$. Considering that the expression levels of plasma miRNAs may be affected by atherosclerosis, hypertension, diabetes, and hyperlipaemia [10], patients with stable angina and without significant coronary lesions requiring percutaneous coronary intervention ( $>50 \%$ stenosis determined by angiography) [22] and $\mathrm{HF}$, who often have similar risk factor baselines for coronary artery disease as patients with prior MI, were enrolled as controls. Demographic, clinical, and echocardiographic characteristics were abstracted from the electronic medical records. Patients were excluded from the current study when they were known to have a recent acute MI, autoimmune disease, infection, cardiogenic shock, valvular disease, severe renal insufficiency (eGFR $<30 \mathrm{ml} / \mathrm{min} / 1.73 \mathrm{~m}^{2}$ ), malignancy, or pregnancy. Baseline characteristics were compared via chi-squared test (categorical variables), two-independentsample $t$-test (normally distributed variables), or MannWhitney $U$ test (nonnormally distributed variables). The present study was approved by the Ethical Review Board of the China-Japan Union Hospital of Jilin University, China. Plasma samples were obtained with informed consent for the proper secondary use of human samples at Jilin Provincial Molecular Biology Research Center for Precision Medicine of Major Cardiovascular Disease. All procedures, including blood storage and data collection were performed in accordance with the institutional guidelines for the use of human samples by China-Japan Union Hospital of Jilin University.

2.3. Echocardiography. Rats were mildly anaesthetized using $3 \%$ isoflurane, and transthoracic echocardiography was performed using a Vivid-i echocardiography machine (General Electric Company, Fairfield, CT, USA) equipped with an 11.5 MHz transducer. Electrocardiographic assessments for patients were performed by three cardiologists who were blinded to the clinical data.

2.4. Pathology. Histologic studies were conducted with $4 \%$ paraformaldehyde-fixed and paraffin-embedded left ventricular samples from rats of all groups. Haematoxylin/eosin staining and Masson's trichrome staining were performed on cross sections of the left ventricle. Images were acquired with a microscope (Olympus Corporation, Tokyo, Japan) equipped with a digital camera and cellSens Dimension software (version 1.16; Olympus Corporation, Tokyo, Japan). 
2.5. ELISA Assay for BNP. The plasma levels of BNP in rats were measured by an ELISA assay (R\&D Systems, Inc., Minneapolis, MN, USA) following the manufacturer's instructions. The optical density values of samples were detected by a spectrophotometer with a wavelength of $450 \mathrm{~nm}$. The normalization curve was obtained from a series of BNP standards provided in the kit.

2.6. RNA Extraction. Total plasma RNA extraction was performed using TRIzol LS solution (Invitrogen; Thermo Fisher Scientific, Inc., Waltham, MA, USA) with a Caenorhabditis elegans (C. elegans) RNA template as a spike-in control miRNA for normalization. Total RNA from tissues and PBMCs of rats was extracted using a TRIzol solution (Invitrogen; Thermo Fisher Scientific, Inc.).

2.7. RNA Sequencing and Bioinformatic Analysis. Following the RNA integrity and concentration examination, $2.5 \mathrm{ng}$ of RNA per plasma sample and $1 \mu \mathrm{g}$ of RNA per myocardial sample were used to prepare the library for small RNAs and messenger RNAs (mRNAs), respectively. Differential expression analysis for miRNAs or mRNAs between the 8week post-MI group and the sham group was conducted using the DESeq package (version 1.10.1). miRNAs or mRNAs with a fold change $(\mathrm{FC})<0.5$ or $>2$ were determined to be differentially expressed. The target genes of miRNAs were predicted using miRWalk (version 3.0), miRanda (version 3.3a), RNAhybrid (version 2.1.1), and TargetScan (version 6.2) analyses. The functions of the target genes were annotated using KOBAS (version 3.0) based on the Kyoto Encyclopedia of Genes and Genomes (KEGG) and the Gene Ontology (GO) databases.

2.8. Real-Time PCR. An aliquot of $3.8 \mu \mathrm{l}$ of RNA was reverse-transcribed to cDNA in a $10 \mu \mathrm{l}$ reaction using a Mir-X First-Strand Synthesis Kit (Takara Bio; Clontech Laboratories, Inc., Mountain View, CA, USA). Both miRNAs and mRNAs were polyadenylated, reverse-transcribed, and quantified using a Mir-X RT-qPCR kit (Takara Bio; Clontech Laboratories, Inc.). The expression levels of miRNAs were normalized to the expression levels of C. elegans miRNA-39 for plasma samples and U6 for tissue samples. The expression levels of mRNAs were normalized to the expression levels of $\alpha$-actin. Specific primers for quantitative PCR were used as follows: DNA-damage-inducible transcript 4 (Ddit4) forward primer, $5^{\prime}$-GCTCTGGACCCCAG TCTAGT-3 ${ }^{\prime}$; Ddit4 reverse primer, $5^{\prime}$-GGGACAGTCCT TCAGTCCTT- ${ }^{\prime}$; sestrin 1 (Sesn 1 ) forward primer, $5^{\prime}$-AA GTGAGGTGGGAGGGTCTGTG-3'; Sesn 1 reverse primer, $5^{\prime}$-TTTGAAAGCCCGTCCGAAGGTC- ${ }^{\prime}$; NF- $\kappa$ B inhibitor $\alpha$ (Nfkbia) forward primer, $5^{\prime}$-GTCTGAACTCGCCACC CAACTG-3'; Nfkbia reverse primer, $5^{\prime}$-GTCCACCAACC GCTCCTTCTTG-3'; $\alpha$-actin forward primer, $5^{\prime}$-GGGTAT GGGTCAGAAGGACT- $3^{\prime}$; and $\alpha$-actin reverse primer, $5^{\prime}$ GAGGCATACAGGGACAACAC- $3^{\prime}$. The entire sequence of mature miRNA with all U's converted to T's was used as a forward primer for each miRNA. The reverse primer was provided in the kit and was complementary to the adapter sequence of the reverse-transcribed primer.

2.9. Exosome Isolation. The isolation of plasma exosomes was supported by a Total Exosome Isolation Kit (Invitrogen; Thermo Fisher Scientific, Inc.) following the manufacturer's instructions. Proteinase $\mathrm{K}$ was used to remove the bulk of protein from the plasma. The exosome-containing pellet was resuspended in PBS. The morphology of the exosomes was observed by transmission electron microscopy. The exosomal markers were examined by western blot analysis. Exosome samples were homogenized in ice-cold RIPA buffer (consisting of $50 \mathrm{mM}$ Tris ( $\mathrm{pH} 7.4$ ), $150 \mathrm{mM} \mathrm{NaCl}, 1 \% \mathrm{NP}$ $40,0.5 \%$ sodium deoxycholate, and $0.1 \%$ sodium dodecyl sulfate) supplemented with protease inhibitors (Beyotime Biotechnology, Shanghai, China). The total protein was loaded for resolution by sodium dodecyl sulfate-polyacrylamide gels under reducing conditions. The primary antibodies against apoptosis-linked gene 2-interacting protein X (ALIX; 1:2000; cat. no. ab186429), tumour susceptibility gene 101 protein (TSG101; $1: 2000$; cat. no. ab125011), and cluster of differentiation 63 (CD63; 1:1000; cat. no. ab108950) were purchased from Abcam (Cambridge, MA, USA). The HRPconjugated secondary antibody (1:5000; cat. no. BS13278) was purchased from Bioworld Technology, Inc. (Louis Park, MN, USA).

2.10. Statistical Analysis. All measurement data are expressed as the means \pm S.E.M. Statistical analysis was performed with PASW Statistics 18 software (version 18.0.0; IBM, Inc., Armonk, NY, USA). Differences between groups were tested by a two-independent-sample $t$-test. Statistical graphs were generated using GraphPad Prism software (version 7.04; GraphPad Software, Inc., San Diego, CA, USA). Receiver operator characteristic (ROC) curves and the area under the curve (AUC) were calculated using MedCalc software (version 18.11; MEDCALC, Inc., Ostend, Belgium). For all analyses, a $P$ value $<0.05$ was considered significantly different.

\section{Results}

3.1. General Characteristics of Postinfarction HF Rats. At 4 weeks post-MI, a reduction in the echocardiographic ejection fraction and fractional shortening measurements and an increase in the plasma levels of BNP were observed in postMI rats (4-week post-MI group) compared with sham rats, indicating damage to cardiac function and the initiation of chronic HF (Figure 1(a)). Over time, cardiac function progressively deteriorated at 8 weeks post-MI (8-week post-MI group) and was dramatically damaged at 12 weeks post-MI (12-week post-MI group) (Figure 1(a)).

Echocardiographic left ventricular internal end diastolic dimension (LVIDd) and left ventricular internal end systolic dimension (LVIDs) values were significantly increased from 4 weeks post-MI to 8 weeks post-MI and mildly increased from 8 weeks post-MI to 12 weeks post-MI (Figure 1(a)). Progressively increased extracellular matrix synthesis and cardiac fibrosis, as determined by histopathology, were seen 
(A)

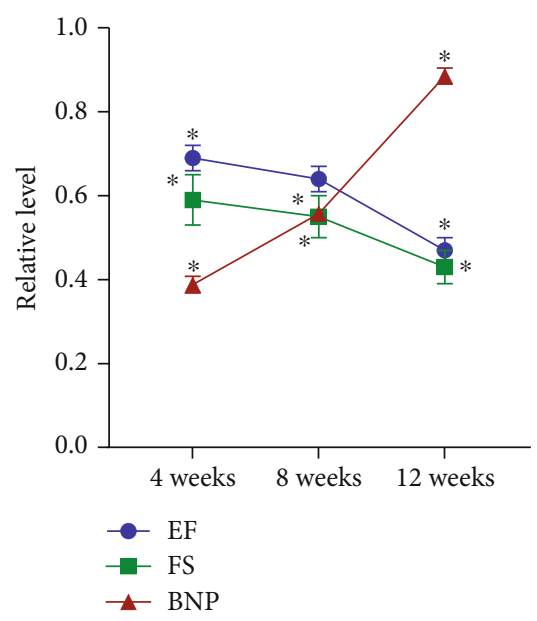

(B)

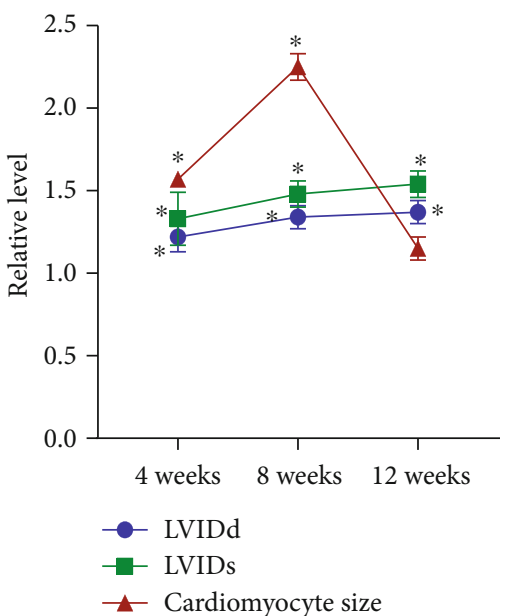

(a)

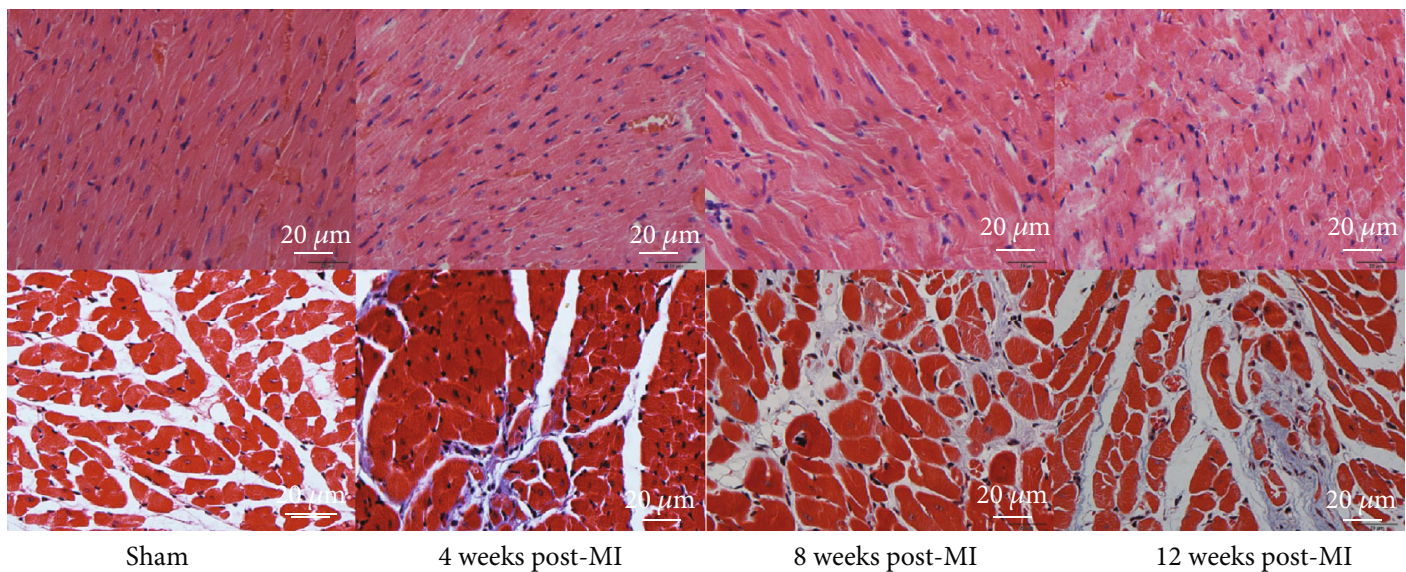

(b)

FIGURE 1: General characteristics of sham and post-MI rats. (a) Echocardiographic EF and FS measurements and plasma BNP levels of sham and post-MI rats at 4 weeks, 8 weeks, and 12 weeks post-MI (A). LVIDd, LVIDs, and cardiomyocyte size values of sham and post-MI rats at 4 weeks, 8 weeks, and 12 weeks post-MI (B). (b) Haematoxylin/eosin staining (upper) and Masson's trichrome staining (lower) of the left ventricle of sham and post-MI rats at 4 weeks, 8 weeks, and 12 weeks post-MI. ${ }^{*} P<0.05$ versus the corresponding sham group. EF: ejection fraction; FS: fractional shortening; BNP: brain natriuretic peptide; MI: myocardial infarction; LVIDd: left ventricular internal end diastolic dimension; LVIDs: left ventricular internal end systolic dimension.

in the left ventricle of post-MI rats over time, which indicated the development of cardiac remodeling (Figure 1(b)). Furthermore, we found that the cardiomyocyte size in an area distant from the site of infarction was larger in the 4-week post-MI and 8-week post-MI groups than in the corresponding sham groups, and this cardiomyocyte hypertrophy was attenuated at 12 weeks post-MI (Figure 1(b)). The dilatation of the left ventricle, the activation of cardiac fibrosis, and cardiomyocyte hypertrophy may be compensatory mechanisms triggered to maintain cardiac function at 4 and 8 weeks post-MI. However, these mechanisms became maladaptive at 12 weeks post-MI and led to further left ventricular dilatation and thinning and significant deterioration in cardiac function.

3.2. Discovery of Candidate Plasma miRNAs. We first analysed the global plasma miRNA expression in the 8-week
post-MI group and corresponding sham group. We detected 619 known miRNAs in the plasma samples. Differential expression analysis indicated that 143 miRNAs were differentially expressed in the 8 -week post-MI group compared with the sham group $(\mathrm{FC}<0.5$ or $>2 ; P<0.05)$. Of the 143 differentially expressed miRNAs, 138 were upregulated and 5 were downregulated in the 8 -week post-MI group compared with the sham group. Hierarchical clustering analysis indicated that differentially expressed miRNAs could clearly distinguish the 8-week post-MI group from the sham group (Figure 2). From the 143 differentially expressed miRNAs, we selected 13 miRNAs based on their FCs and probability values for further study.

3.3. Validation of Candidate Plasma miRNAs. We validated the expression levels of 13 candidate miRNAs in the 8-week post-MI group and sham group. In the 8 -week post-MI 


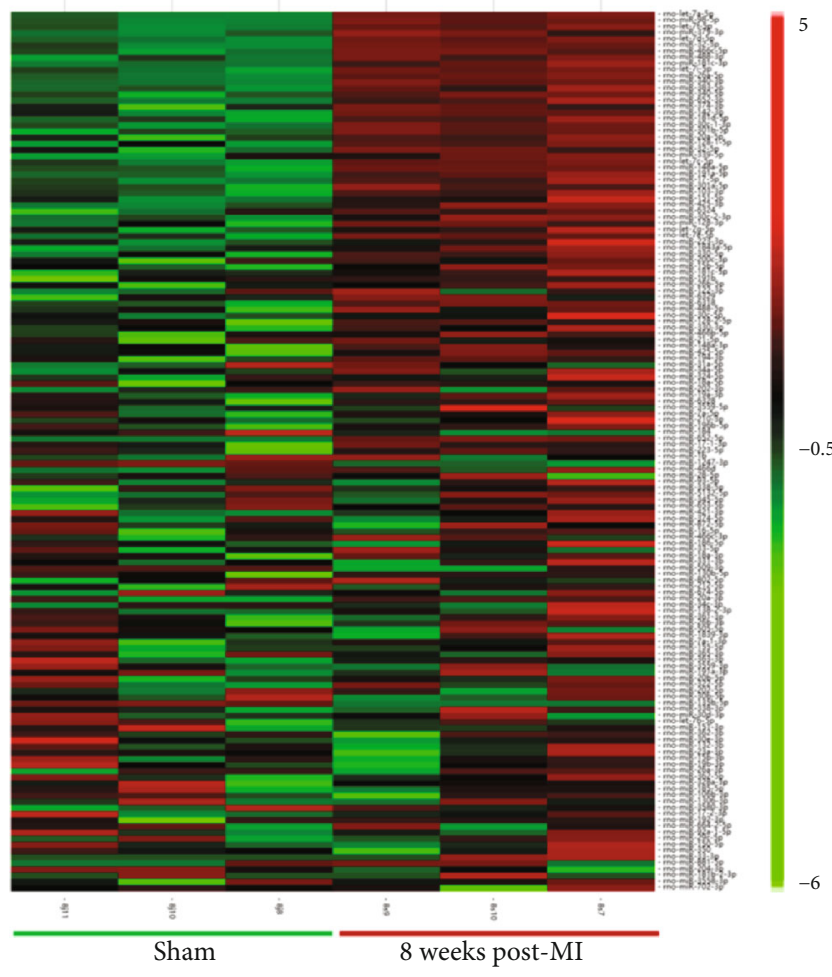

Figure 2: Hierarchical clustering of 143 DE circulating miRNAs $(\mathrm{FC}<0.5$ or $>2 ; P<0.05)$ in the 8 -week post-MI group $(n=3)$ compared with the sham group $(n=3)$. DE: differentially expressed; FC: fold change.

group, rats had an ejection fraction lower than $50 \%$. In the sham group, rats had an ejection fraction higher than $70 \%$. The FCs of the plasma miRNA levels in the 8-week postMI group versus the sham group are shown in Figure 3(a). Of the 13 miRNAs that were significantly upregulated in miRNA sequencing, 5 miRNAs were also significantly upregulated in the validated 8-week post-MI group compared with the sham group. The expression levels of miR-20a-5p, miR$340-5 p$, and let-7i-5p were increased more than threefold in the 8 -week post-MI group compared with the sham group. In addition, we evaluated the accuracy and sensitivity of miR-20a-5p, miR-340-5p, and let-7i-5p for predicting $\mathrm{HF}$ in the 8 -week post-MI group and the sham group using a ROC curve and the AUC. The AUCs of miR-20a-5p, miR340-5p and let-7i-5p were $0.863(P<0.05), 0.883(P<0.05)$, and $0.892(P<0.05)$, respectively (Figure $3(\mathrm{~b}))$. The expression levels of miR-20a-5p, miR-340-5p, and let-7i-5p were closely associated with HF.

3.4. Temporal Expression of miRNAs in Plasma and Myocardium from 4 Weeks Post-MI to 12 Weeks Post-MI. We evaluated the expression levels of miR-20a-5p, miR$340-5 p$, and let-7i-5p in plasma and myocardium of the post-MI rat cohort. The expression levels of miR-20a-5p, miR-340-5p, and let-7i-5p showed an upward trend from 4 weeks to 8 weeks post-MI, whereas their expression dramatically decreased to baseline levels at 12 weeks post-MI, and the expression levels of miR-20a-5p were significantly decreased in the 12-week post-MI group compared with the sham group (Figure 4). As shown in Table 1, there were significant positive correlations between the expression levels of miR-20a-5p, miR-340-5p, and let-7i-5p and LVIDd, LVIDs, and cardiomyocyte size at 4 weeks and 8 weeks post-MI. Additionally, inverse correlations were identified between the expression levels of miR-20a-5p and LVIDd and LVIDs at 12 weeks post-MI.

The expression levels of miR-20a-5p and let-7i-5p were elevated in the myocardium of the 4 -week post-MI group compared with the sham group. No significant difference in the expression levels of miR-20a-5p, miR-340-5p, and let$7 \mathrm{i}-5 \mathrm{p}$ in the myocardium was identified between the postMI groups and the sham groups at 8 and 12 weeks post-MI (Figure 4).

3.5. Possible Origins of Plasma miRNAs. We quantified the expression levels of miR-20a-5p, miR-340-5p, and let-7i-5p in the HF-related tissues, including the liver, spleen, lung, kidney, thymus, and PBMCs at 8 weeks post-MI. The expression levels of miR-20a-5p, miR-340$5 p$, and let-7i-5p were upregulated in PBMCs at 8 weeks post-MI (Figure 5(a)), and there was no significant difference in the miR-20a-5p, miR-340-5p, and let-7i-5p levels in the liver, spleen, lung, kidney, and thymus tissues between the 8 -week post-MI group and the sham group (see Figure S1 in the Supplementary Material for quantitative results). We subsequently investigated the temporal expression of miR20a-5p, miR-340-5p, and let-7i-5p in PBMCs at 4 weeks and 12 weeks post-MI. The expression levels of miR-20a$5 \mathrm{p}$ and miR-340-5p in PBMCs showed an upward trend that was similar to the trend in plasma from 4 weeks to 8 weeks post-MI (Figure 5(a)). These results indicated that PBMCs may be a potential origin of plasma miR-20a-5p and miR-340-5p. Furthermore, exosomes were isolated from plasma (Figure 5(b)) and used to evaluate the expression levels of exosome-original miR-20a-5p, miR-340-5p, and let$7 \mathrm{i}-5 \mathrm{p}$. Only the expression levels of miR-20a-5p showed a significant 1.7 -fold increase in the 8 -week post-MI group compared with the sham group, indicating that plasma miR20a-5p may be partly transported by exosomes (Figure 5(c)).

3.6. Target $m R N A$ s of $m i R-20 a-5 p$, $m i R-340-5 p$, and let-7i-5p. Plasma miR-20a-5p, miR-340-5p, and let-7i-5p may play important roles in the development of left ventricular remodeling. Since miRNAs are known to regulate gene expression via the degradation or translational repression of their target mRNAs [5], a parallel miRNA-mRNA expression profiling approach $[23,24]$ was used to identify potential mRNA targets of miR-20a-5p, miR-340-5p, and let-7i-5p. The global mRNA expression in the myocardium (an area distant from the site of infarction) of rats in the 8-week post-MI group and the corresponding sham group was analysed using mRNA sequencing. Differential expression analysis indicated that 36 mRNAs were differentially expressed in the 8-week post-MI group compared with the sham group $(\mathrm{FC}<0.5$ or $>2 ; P<0.05)$. Of the 36 differentially expressed mRNAs, 10 were upregulated and 26 were downregulated in the 

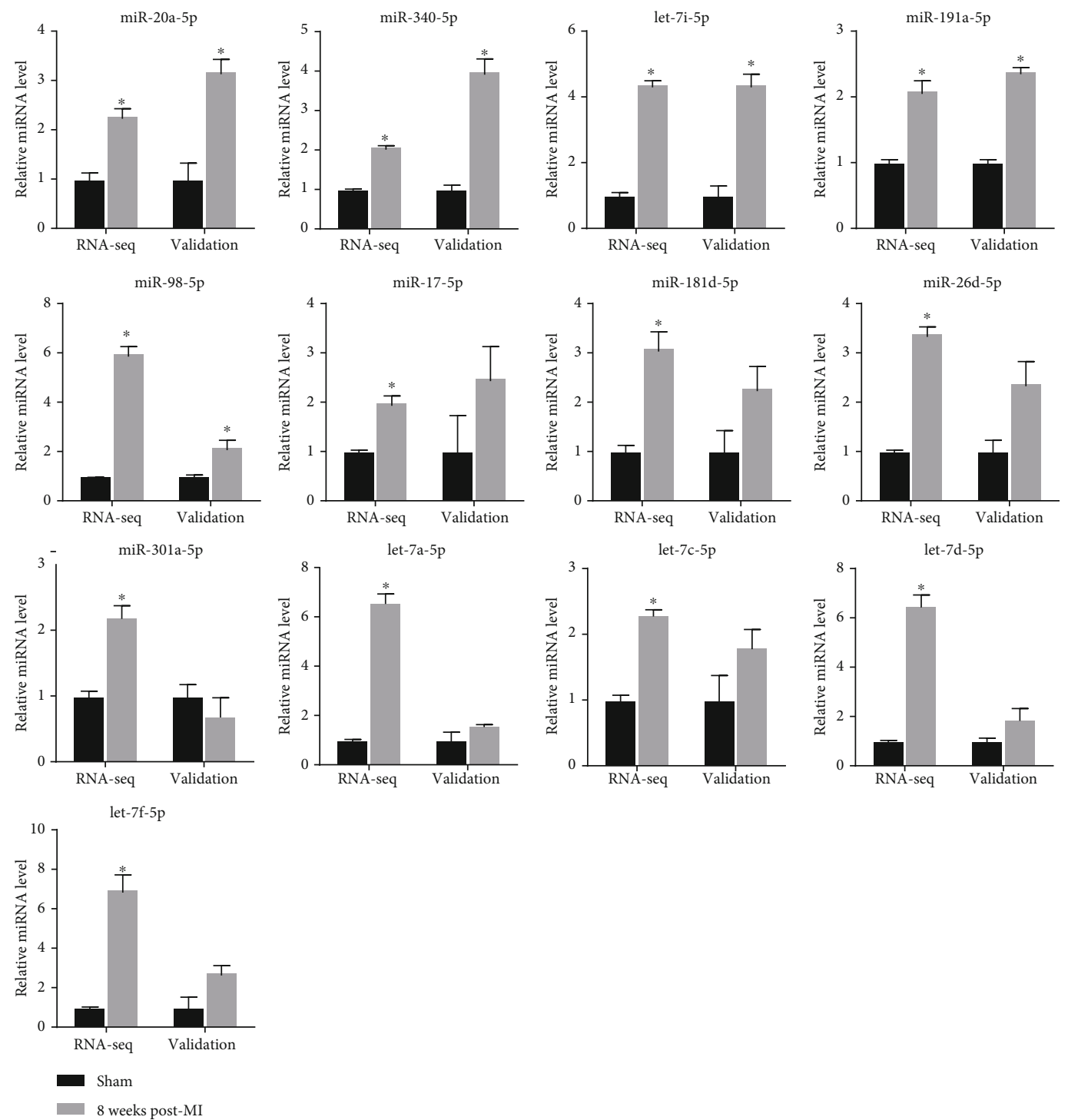

(a)
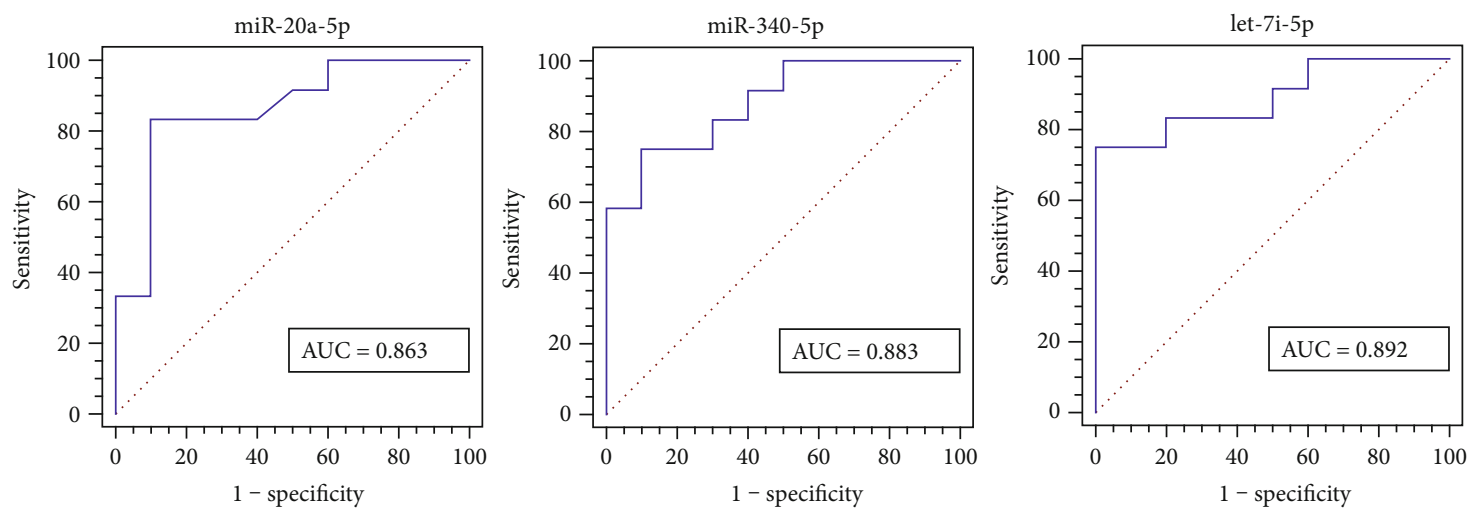

(b)

FIGURE 3: Validation of candidate plasma miRNAs. (a) Expression levels of 13 candidate plasma miRNAs in the 8-week post-MI group and the sham group. The left 2 bars of each panel show miRNA levels detected by miRNA sequencing in the 8 -week post-MI group $(n=3)$ and the sham group $(n=3)$. The right 2 bars of each panel show the same miRNA levels validated using real-time PCR in the 8-week post-MI group $(n=16)$ and the sham group $(n=12)$. (b) ROC curves and the AUCs of miR-20a-5p, miR-340-5p, and let-7i-5p for predicting HF at 8 weeks post-MI. ${ }^{*} P<0.05$ versus the corresponding sham group. RNA-seq: RNA sequencing; MI: myocardial infarction; HF: heart failure; ROC: receiver operator characteristic; AUC: area under the curve. 

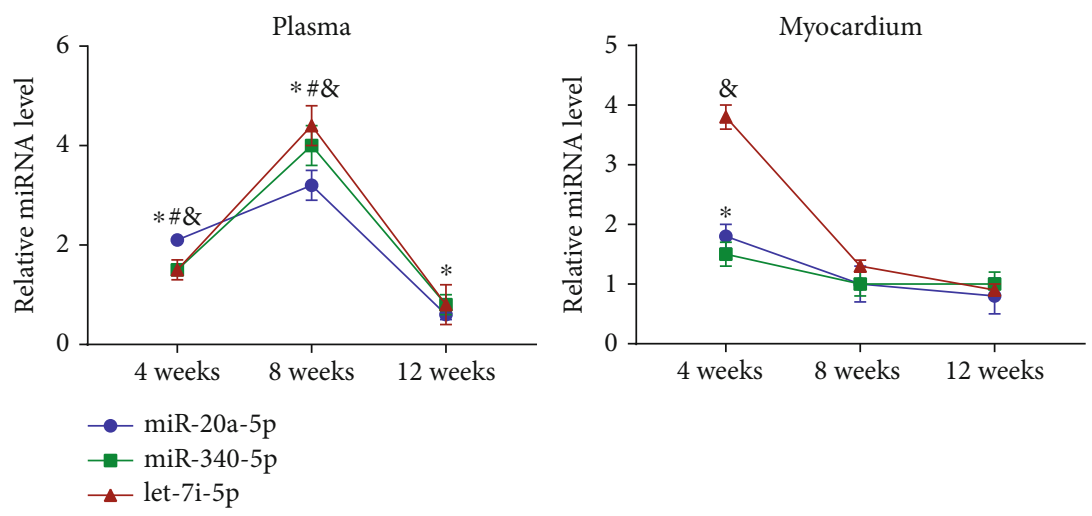

FIGURE 4: Temporal expression of miR-20a-5p, miR-340-5p, and let-7i-5p in the plasma and myocardium of post-MI rats from 4 weeks postMI to 12 weeks post-MI. ${ }^{*} P<0.05$ versus the corresponding sham group (miR-20a-5p); ${ }^{\#} P<0.05$ versus the corresponding sham group (miR-340-5p); ${ }^{8} P<0.05$ versus the corresponding sham group (let-7i-5p). MI: myocardial infarction.

TABLE 1: Correlations between the expression levels of plasma miRNAs and LVIDd, LVIDs, and cardiomyocyte size at 4 weeks, 8 weeks, and 12 weeks post-MI.

\begin{tabular}{lccccccccc}
\hline & & LVIDd & & & \multicolumn{2}{c}{ LVIDs } & \multicolumn{3}{c}{ Cardiomyocyte size } \\
& 4 weeks & 8 weeks & 12 weeks & 4 weeks & 8 weeks & 12 weeks & 4 weeks & 8 weeks & 12 weeks \\
\hline \multirow{2}{*}{ miR-20a-5p } & $r=0.793^{*}$ & $r=0.889^{*}$ & $r=-0.816^{*}$ & $r=0.783^{*}$ & $r=0.861^{*}$ & $r=-0.735^{*}$ & $r=0.977^{*}$ & $r=0.932^{*}$ & $r=-0.609$ \\
& $P=0.019$ & $P=0.003$ & $P=0.013$ & $P=0.022$ & $P=0.006$ & $P=0.038$ & $P=0$ & $P=0.01$ & $P=0.109$ \\
miR-340-5p & $r=0.863^{*}$ & $r=0.733^{*}$ & $r=-0.393$ & $r=0.855^{*}$ & $r=0.707^{*}$ & $r=-0.434$ & $r=0.848^{*}$ & $r=0.74^{*}$ & $r=-0.386$ \\
& $P=0.006$ & $P=0.039$ & $P=0.336$ & $P=0.007$ & $P=0.049$ & $P=0.283$ & $P=0.008$ & $P=0.036$ & $P=0.345$ \\
& $r=0.754^{*}$ & $r=0.829^{*}$ & $r=-0.061$ & $r=0696^{*}$ & $r=0.799^{*}$ & $r=-0.302$ & $r=0.719^{*}$ & $r=0.868^{*}$ & $r=-0.009$ \\
let-7i-5p & $P=0.031$ & $P=0.011$ & $P=0.887$ & $P=0.019$ & $P=0.017$ & $P=0.468$ & $P=0.044$ & $P=0.005$ & $P=0.984$ \\
\hline
\end{tabular}

MI: myocardial infarction; $r$ : Pearson correlation coefficient; LVIDd: left ventricular internal end diastolic dimension; LVIDs: left ventricular internal end systolic dimension; MI: myocardial infarction. ${ }^{*} P<0.05$ versus the corresponding sham group.

8-week post-MI group compared with the sham group. Differentially expressed mRNAs clearly distinguished the 8week post-MI group from the sham group (Figure 6(a)). The target genes of miR-20a-5p, miR-340-5p, and let-7i-5p were predicted using miRWalk, miRanda, RNAhybrid, and TargetScan analyses and subsequently paired to the downregulated mRNAs generated from mRNA sequencing (Figure 6(b)). The symbols of paired genes are presented in Figure 6(c) (19 genes in total). There was no paired gene between the targets of miR-340-5p and the downregulated myocardial mRNAs. However, there were 671 target genes of miR-340-5p in common with the target genes of miR20a-5p and/or let-7i-5p. Additionally, several pathways mechanistically associated with cardiac remodeling were presented in the KEGG annotation results of the 19 common target genes, including the mechanistic target of rapamycin (mTOR), nuclear factor- (NF-) $\kappa \mathrm{B}$, tumour necrosis factor (TNF), apoptosis, p53, and other signaling pathways (see Table S1 in Supplementary Materials for comprehensive KEGG analysis). From the 19 common genes, Ddit4, Sesn1, and Nfkbia were selected for further validation with realtime PCR in the 8-week post-MI group and the sham group. These 3 genes play roles in the cellular processes, including cellular growth, autophagy, apoptosis, inflammation, and fibrosis [25-29], which are associated with the progression of cardiac remodeling [30]. As presented in Figure 6(d), the expression levels of Ddit4, Sesn1, and Nfkbia mRNAs were also significantly reduced in the validated 8 -week post-MI group compared with the corresponding sham group. Ddit4, Sesn1, and Nfkbia may be potential target genes of miR-20a$5 \mathrm{p}$, and Ddit4 may be a potential target gene of let-7i-5p.

\subsection{Evaluation of Candidate Plasma miRNAs as Biomarkers} for Postinfarction Left Ventricular Remodeling in Patient Population. The sequences of miR-20a-5p, miR-340-5p, and let-7i-5p share significant homology between rats and humans. The expression levels of these 3 miRNAs were subsequently investigated in 32 patients with postinfarction $\mathrm{HF}$ and 16 control patients with stable angina and without significant coronary lesions and HF. The baseline characteristics of the subjects are presented in Table 2. The echocardiographic ejection fraction measurements of patients with postinfarction $\mathrm{HF}$ were lower than $60 \%$ and higher than $21 \%$. The levels of NT-proBNP in patients with postinfarction $\mathrm{HF}$ were higher than $125 \mathrm{pg} / \mathrm{ml}$. Additionally, $56.3 \%$ and $62.5 \%$ patients with postinfarction $\mathrm{HF}$ used $\beta$-receptor blockers and angiotensin-converting enzyme inhibitors/angiotensin receptor blockers, respectively, to treat postinfarction left ventricular remodeling and/or hypertension. No significant difference in the echocardiographic measurements of the interventricular septum and left ventricular posterior wall was identified between patients with postinfarction HF and 


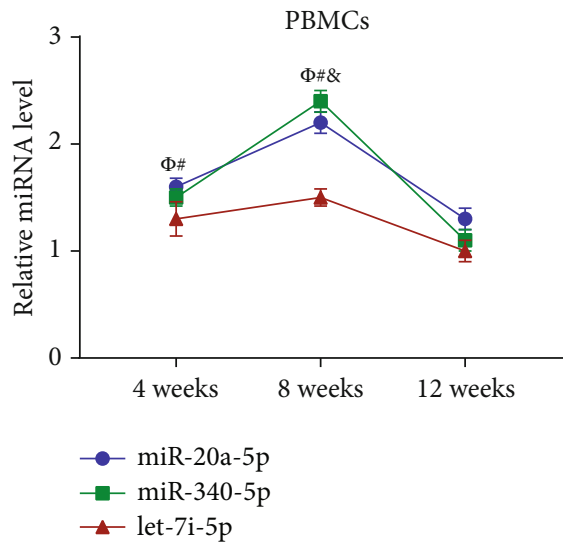

(a)
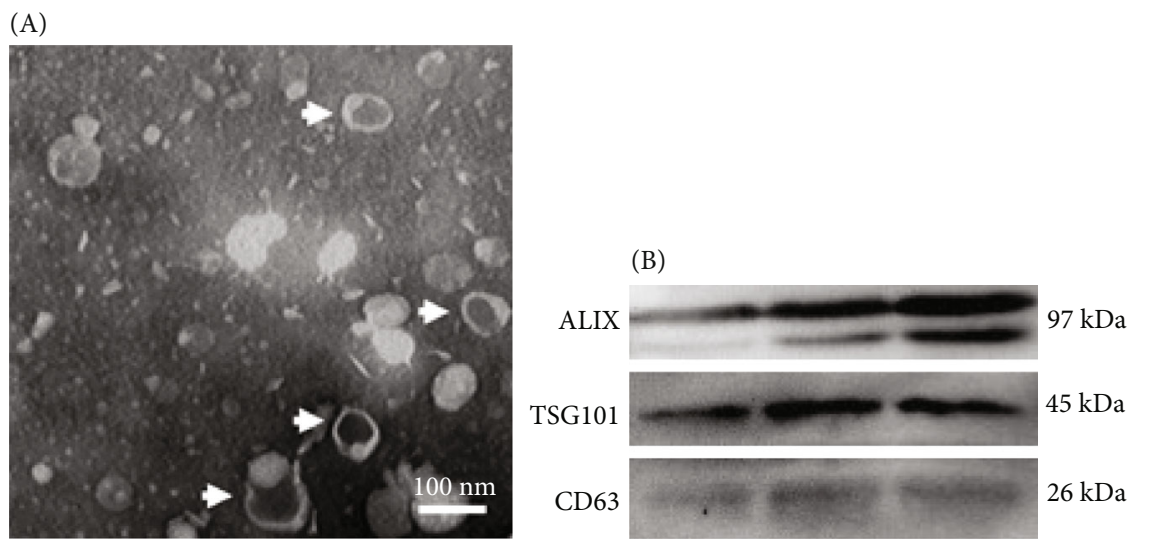

(b)
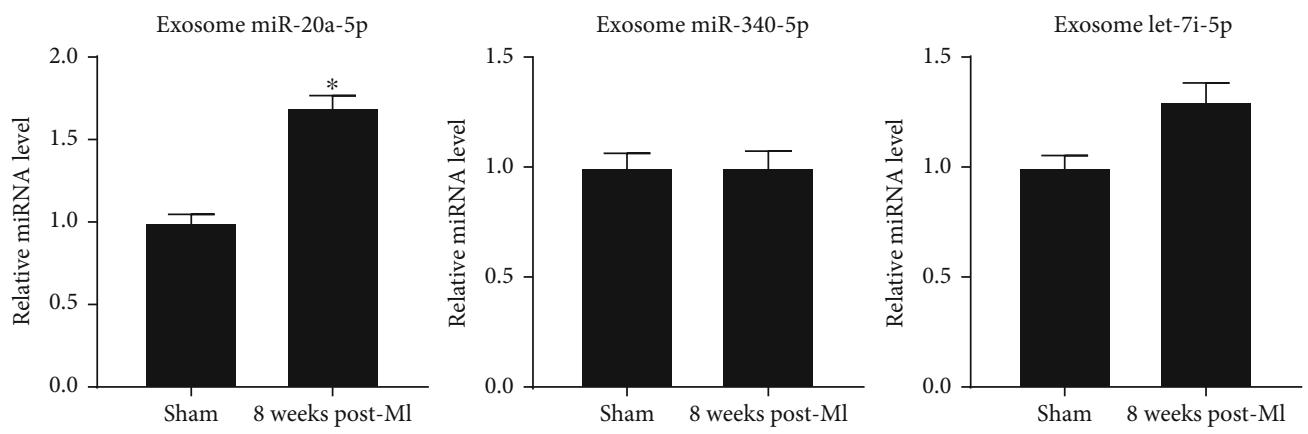

(c)

FIgURe 5: Possible origins of plasma miR-20a-5p, miR-340-5p, and let-7i-5p. (a) The temporal expression of miR-20a-5p, miR-340-5p, and let-7i-5p in the PBMCs of post-MI rats and sham controls at 4, 8, and 12 weeks post-MI. (b) Transmission electron microscope image of exosomes isolated from plasma (A; white arrow) and western blot of exosomal markers ALIX, TSG101, and CD63 (B). (c) Expression levels of exosome-original miR-20a-5p, miR-340-5p, and let-7i-5p at 8 weeks post-MI. ${ }^{*} P<0.05$ versus the corresponding sham group; ${ }^{\Phi} P<0.05$ versus the corresponding sham group (miR-20a-5p); ${ }^{\#} P<0.05$ versus the corresponding sham group (miR-340-5p); ${ }^{\&} P<0.05$ versus the corresponding sham group (let-7i-5p). MI: myocardial infarction; PBMCs: peripheral blood mononuclear cells; ALIX: apoptosis-linked gene 2-interacting protein X; TSG101: tumour susceptibility gene 101 protein; CD63: cluster of differentiation 63.

controls. The plasma levels of miR-20a-5p, miR-340-5p, and let-7i-5p were significantly increased in patients with postinfarction HF compared with controls (Figure 7(a)). Additionally, the AUCs of miR-20a-5p, miR-340-5p, and let-7i-5p were $0.867(P<0.05), 0.825(P<0.05)$, and $0.779(P<0.05)$, respectively (Figure $7(\mathrm{~b})$ ). However, only the expression levels of miR-20a-5p presented significant positive correlations with LVIDd (Pearson correlation coefficient: $0.435 ; P$ value: 0.002 ) and left ventricular end diastolic volume (LVEDV; Pearson correlation coefficient: 0.323 ; $P$ value: 0.025 ) (Table 3 ).

\section{Discussion}

Following MI, progressive cardiac remodeling is associated with cardiac dysfunction and poor prognosis for survival [31]. During the development of left ventricular remodeling, 


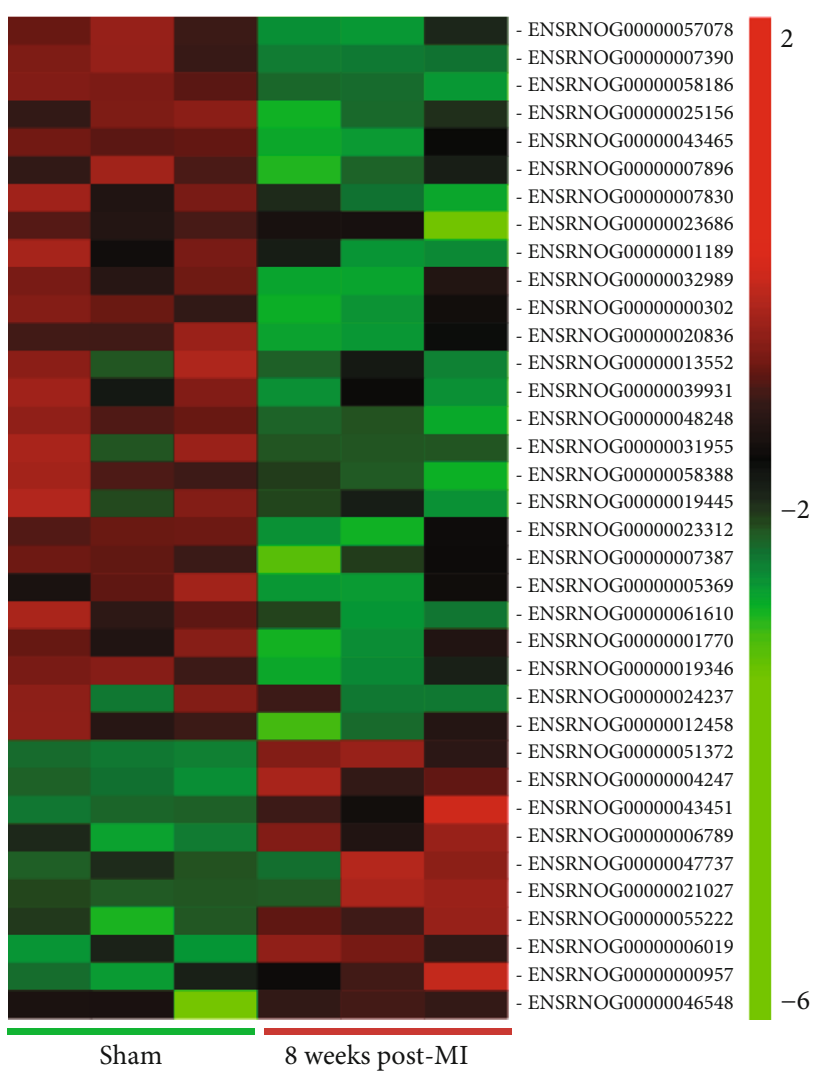

(a)

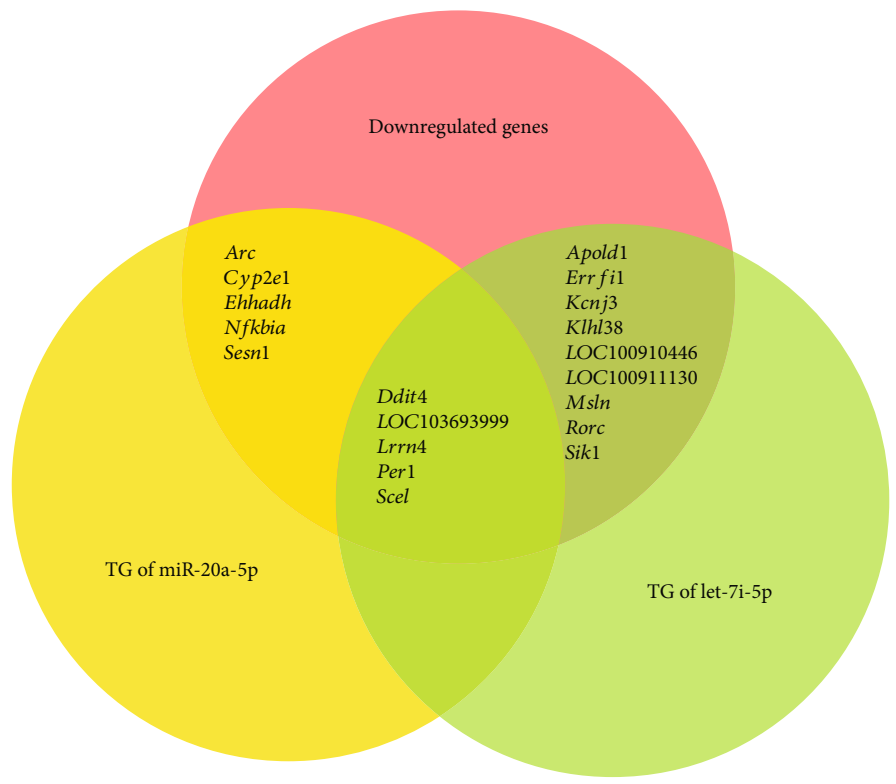

(c)
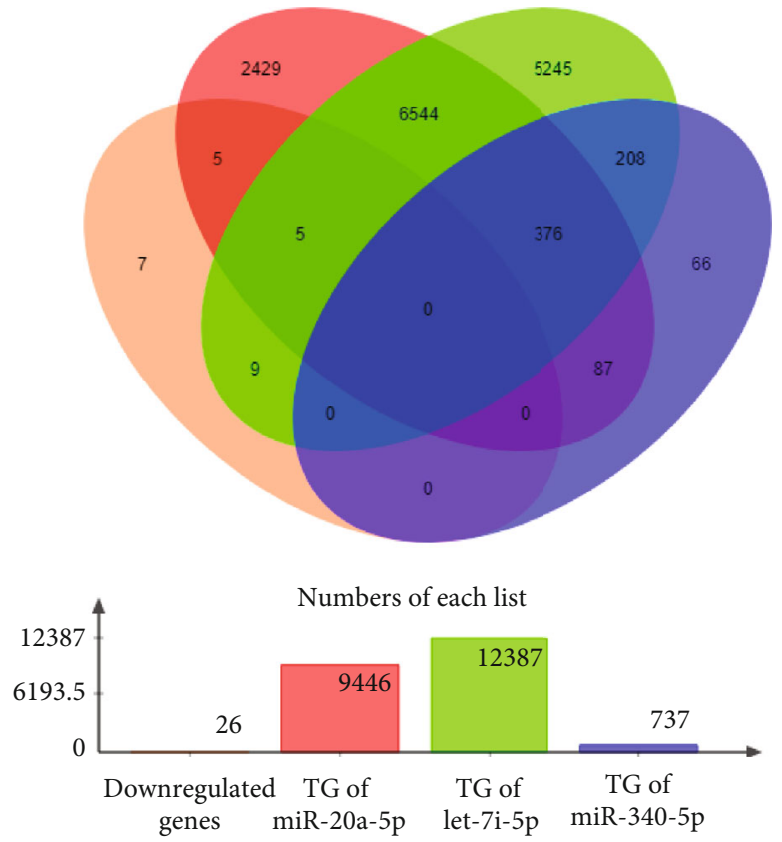

(b)

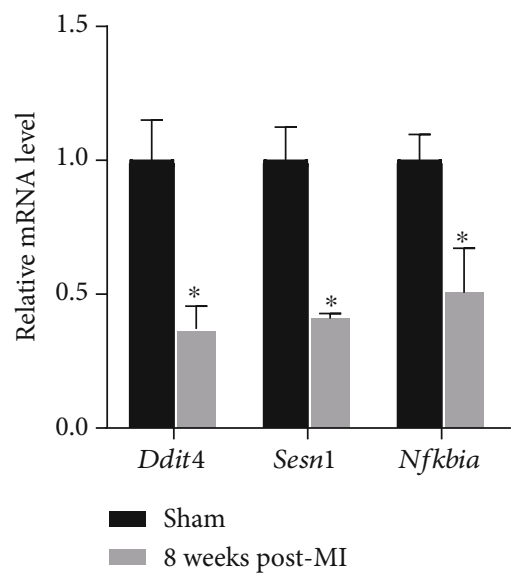

(d)

Figure 6: Target genes of plasma miR-20a-5p, miR-340-5p, and let-7i-5p. (a) Hierarchical clustering of 36 DE mRNAs (FC $<0.5$ or $>2$; $P<0.05)$ in the 8 -week post-MI group $(n=3)$ compared with the sham group $(n=3)$. (b) The number and overlap among the downregulated mRNAs generated from mRNA sequencing and the predicted target genes of miR-20a-5p, miR-340-5p, and let-7i-5p. (c) The common genes among the downregulated genes, the target genes of miR-20a-5p, and the target genes of let-7i-5p. (d) The expression levels of Ddit4, Sesn 1, and Nfkbia mRNAs in the validated 8 -week post-MI group $(n=4)$ compared with the corresponding sham group $(n=4)$, which were examined with real-time PCR. ${ }^{*} P<0.05$ versus the sham group. DE: differentially expressed; mRNA: messenger RNA; MI: myocardial infarction; FC: fold change; TG: target genes; Ddit4: DNA-damage-inducible transcript 4; Sesn1: sestrin 1; Nfkbia: NF- $\kappa$ B inhibitor $\alpha$. 
TABLE 2: Baseline characteristics of the subjects.

\begin{tabular}{|c|c|c|}
\hline Variable & Control $(n=16)$ & Postinfarction HF $(n=32)$ \\
\hline Age (years) ${ }^{\mathrm{I}}$ & $61.1(12.4)$ & $62.4(10.2)$ \\
\hline Female & $9(56.3)$ & $17(53.1)$ \\
\hline Diabetes & $4(25.0)$ & $10(31.3)$ \\
\hline Hypertension & $8(50.0)$ & $15(46.9)$ \\
\hline Current smoker & $3(18.8)$ & $5(15.6)$ \\
\hline Triglycerides $(\mathrm{mmol} / \mathrm{l})^{\mathrm{I}}$ & $1.92(1.62)$ & $1.58(1.02)$ \\
\hline Total cholesterol $(\mathrm{mmol} / \mathrm{l})^{\mathrm{I}}$ & $4.47(1.81)$ & $4.00(1.00)$ \\
\hline $\mathrm{LDL}(\mathrm{mmol} / \mathrm{l})^{\mathrm{I}}$ & $2.65(1.13)$ & $2.54(0.89)$ \\
\hline $\mathrm{HDL}(\mathrm{mmol} / \mathrm{l})^{\mathrm{I}}$ & $1.20(0.26)$ & $1.05(0.21)$ \\
\hline Creatinine $(\mu \mathrm{mol} / \mathrm{l})^{\mathrm{I}}$ & $73.47(11.20)$ & $84.47(14.82)$ \\
\hline$\beta$-Receptor blockers & $4(25.0)$ & $18(56.3)^{*}$ \\
\hline ACE inhibitors or AR blockers & $5(31.3)$ & $20(62.5)^{*}$ \\
\hline NT-proBNP $(\mathrm{pg} / \mathrm{ml})^{\S}$ & $99.1(71.5,132.8)$ & $831.0(152.5,1605.0)^{*}$ \\
\hline \multicolumn{3}{|l|}{ Echocardiography } \\
\hline LVIDd $(\mathrm{mm})^{\mathrm{I}}$ & $42.6(2.3)$ & $54.1(7.7)^{*}$ \\
\hline $\operatorname{LVEDV}(\mathrm{ml})^{\mathrm{I}}$ & $105.4(34.6)$ & $159.9(65.4)^{*}$ \\
\hline Ejection fraction $(\%)^{\mathrm{I}}$ & $73.2(3.7)$ & $44.5(9.4)^{*}$ \\
\hline Fractional shortening $(\%)^{\mathrm{I}}$ & $42.0(2.8)$ & $21.3(5.6)^{*}$ \\
\hline $\operatorname{IVS}(\mathrm{mm})^{\mathrm{I}}$ & $9.5(1.9)$ & $10.3(1.6)$ \\
\hline $\operatorname{LVPW}(\mathrm{mm})^{\mathrm{I}}$ & $9.5(1.8)$ & $9.8(1.2)$ \\
\hline
\end{tabular}

${ }^{\mathrm{I}}$ Mean (SEM), no. (\%), and ${ }^{\$}$ median (25\% percentile, $75 \%$ percentile) of nonnormally distributed variables. HF: heart failure; LDL: low-density lipoprotein cholesterol; HDL: high-density lipoprotein cholesterol; ACE: angiotensin-converting enzyme; AR: angiotensin receptor; NT-proBNP: N-terminal pro-B type natriuretic peptide; LVIDd: left ventricular internal end diastolic dimension; LVEDV: left ventricular end diastolic volume; IVS: interventricular septum; LVPW: left ventricular posterior wall. ${ }^{*} P<0.05$ versus controls.

gross pathologic changes in the left ventricular configuration and volume evaluated by echocardiography have been widely used to predict the clinical outcomes of individuals [2]. However, on a molecular level, biomarkers for left ventricular remodeling are limited to proteins that reflect haemodynamic stress (such as natriuretic peptides) or necrosis (such as cardiac troponins) [12]. Recently, miRNAs, a class of evolutionarily conserved small noncoding RNAs, have been discovered to be stably transported in the blood [10]. Since miRNAs are known to function as gene expression repressors at the posttranscriptional level by targeting the corresponding mRNAs and play important roles in the development of $\mathrm{HF}$, circulating miRNAs have been proposed to be promising biomarkers for left ventricular remodeling [5, 32, 33]. However, the temporal and tissue-specific expression of miRNAs following MI may affect the diagnostic specificity and reproducibility of the use of circulating miRNAs as biomarkers for left ventricular remodeling $[11,34]$. In the current study, using a postinfarction HF rat cohort, our data revealed that the expression levels of plasma miR-20a-5p, miR-340-5p, and let-7i-5p gradually increased and were closely associated with left ventricular hypertrophy and dilatation from 4 weeks to 8 weeks post-MI. At 12 weeks post-MI, the mechanisms underlying left ventricular remodeling became maladaptive and led to significant deterioration in cardiac function and further left ventricular dilatation and thinning. The expression levels of plasma miR-340-5p and let-7i-5p were restored to baseline levels, and the expression levels of miR-20a-5p presented significant inverse correlations with the dilatation of the left ventricle at 12 weeks post-MI. The target genes of miR-20a-5p and let-7i-5p were involved in several pathways associated with the development of left ventricular remodeling. The expression levels of miR-20a-5p, miR-340-5p, and let-7i-5p were also temporally regulated in the myocardium and PBMCs. It may be suggested that these 3 miRNAs played functional roles in the pathophysiology of left ventricular remodeling, and the loss of increased miR-20a-5p, miR$340-5 p$, and let-7i-5p was associated with the process of maladaptive left ventricular remodeling. In the clinical study, patients with postinfarction HF presented a wide range of echocardiographic left ventricular phenotypes, and a lack of extreme phenotypes associated with severe left ventricular remodeling, which may be due to the development of left ventricular remodeling, can be affected by different infarct sizes and time points, antiremodeling medical therapy, and myocardial revascularization strategies. Similar to the expression pattern in postinfarction $\mathrm{HF}$ rats at 4 and 8 weeks postMI, the expression levels of plasma miR-20a-5p, miR-340-5p, and let-7i-5p were increased in patients with postinfarction HF. Additionally, the expression levels of miR-20a-5p presented significant correlations with LVIDd and LVEDV. In accordance with our findings, in a community-based observational cohort, the expression levels of miR-20a-5p were demonstrated to be associated with the development of left 

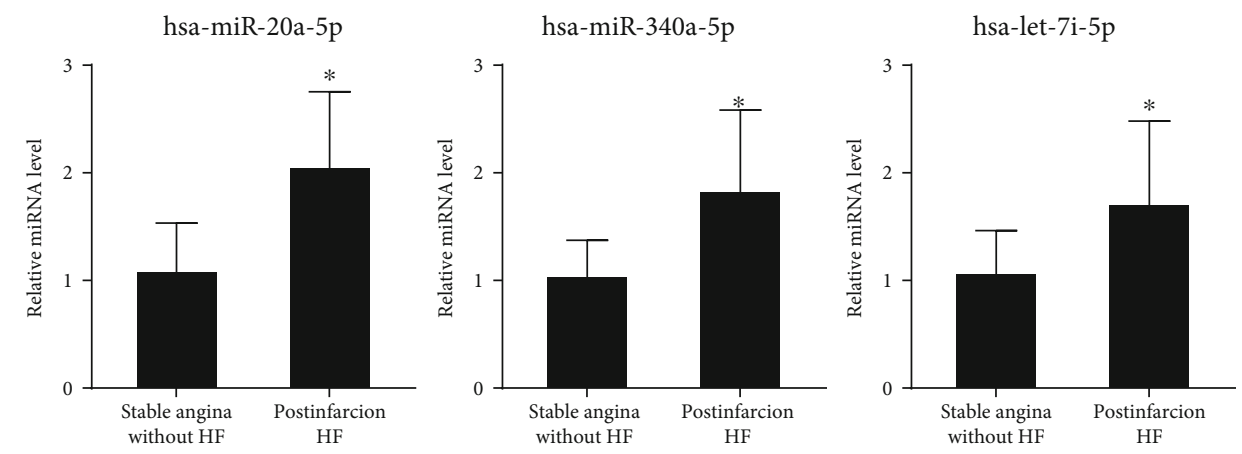

(a)
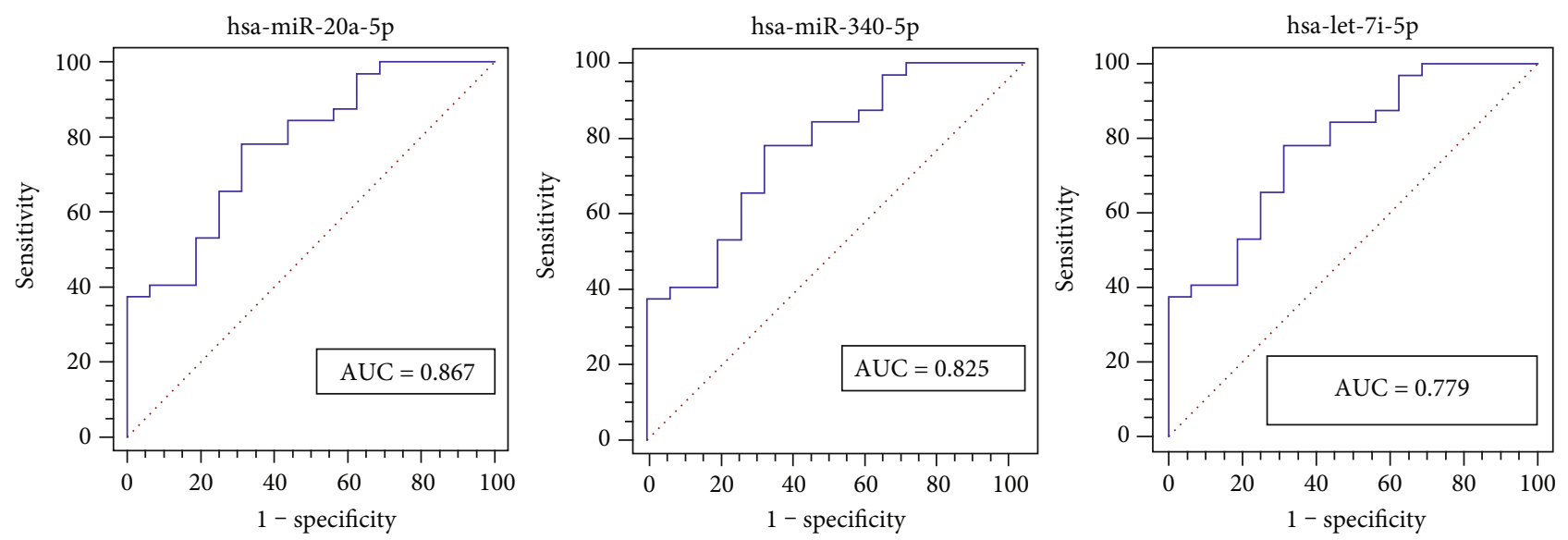

(b)

FIGURE 7: Expression of candidate plasma miRNAs in the patient population. (a) The expression levels of plasma miR-20a-5p, miR-340-5p, and let-7i-5p in patients with postinfarction $\operatorname{HF}(n=32)$ and control patients with stable angina and without significant coronary lesions and HF $(n=16)$. (b) ROC curves and the AUCs of miR-20a-5p, miR-340-5p, and let-7i-5p. ${ }^{*} P<0.05$ versus patients with stable angina and without significant coronary lesions and HF. HF: heart failure; ROC: receiver operator characteristic; AUC: area under the curve.

TABLE 3: Correlations between the expression levels of plasma miRNAs and LVIDd, LVEDV, IVS, and LVPW in the patient population.

\begin{tabular}{lcccc}
\hline & LVIDd & LVEDV & IVS & LVPW \\
\hline hsa-miR-20a-5p & $r=0.435^{*}, P=0.002$ & $r=0.323^{*}, P=0.025$ & $r=0.218, P=0.137$ & $r=0.199, P=0.174$ \\
hsa-miR-340-5p & $r=0.219, P=0.134$ & $r=0.225, P=0.124$ & $r=0.180, P=0.221$ & $r=0.018, P=0.904$ \\
hsa-let-7i-5p & $r=0.200, P=0.173$ & $r=0.223, P=0.128$ & $r=0.062, P=0.675$ & $r=0.090, P=0.544$ \\
\hline
\end{tabular}

HF: heart failure; $r$ : Pearson correlation coefficient; LVIDd: left ventricular internal end diastolic dimension; LVEDV: left ventricular end diastolic volume; IVS: interventricular septum; LVPW: left ventricular posterior wall. ${ }^{*} P<0.05$ versus stable angina patients without significant coronary lesions and HF.

ventricular remodeling and HF incidence during the followup period [35]. Therefore, miR-20a-5p may be a novel potential biomarker for postinfarction left ventricular remodeling.

The results in the present study indicated that miR-20a$5 p$ and let-7i-5p targeted a set of genes involved in the pathways potentially associated with the pathophysiology of left ventricular remodeling, including the mTOR, NF- $\kappa \mathrm{B}$, TNF, apoptosis, and p53 signaling pathways. Among these target genes, the expression levels of Ddit4, Sesn1, and Nfkbia mRNAs, which are closely associated with cellular growth, inflammation, and cell survival, were verified to be reduced in the myocardium of rats at 8 weeks post-MI. DDIT4 is a stress-induced protein and functions as an inhibitor of mTOR kinase [25, 36, 37]. mTOR kinase is an important regulator that promotes cellular growth and proliferation
$[38,39]$. Sesn 1 is a target gene of the tumour suppressor p53, a stress-activated transcription factor that inhibits cellular growth and proliferation [40]. The protein of Nfkbia is an inhibitor of NF- $\kappa \mathrm{B}$ transcription factor, which plays multifaceted roles in the regulation of inflammation and cell survival during the development of cardiac remodeling [41]. Additionally, previous studies supported the progrowth and antiapoptosis effects of miR-20a-5p and let-7i-5p on cardiomyocytes or myoblasts [42-45]. Therefore, Ddit4, Sesn1, and Nfkbia may be potential target genes of miR-20a-5p and/or let-7i-5p.

In patients with acute decompensated HF, the expression levels of plasma let-7i-5p were lower in patients with worsening of renal function compared with patients without worsening of renal function [46]. In a cohort of $\mathrm{HF}$ patients 
with and without atherosclerotic disease, the lower levels of plasma let-7i-5p were associated with the risk of rehospitalizations due to cardiovascular causes during 18 months of followup [45]. In the current study, the loss of increased let-7i-5p was associated with the phenotypes of adverse left ventricular remodeling. It may be suggested that let-7i-5p plays protective roles in the development of HF and the lower circulating let7i-5p levels are associated with the poor prognosis of HF.

In the present study, our data indicated that the expression of miRNAs in plasma, myocardium, and PBMCs may be temporally interconnected. Similarly, in patients with dilated cardiomyopathy, the miRNA expression profile was specifically modulated in PBMCs, and many differentially expressed miRNAs were deregulated in the myocardium in previous studies [47]. Additionally, in an ischemia reperfusion mouse cohort, the expression levels of the majority of candidate miRNAs associated with left ventricular remodeling were upregulated in the myocardium at acute time points and in plasma at chronic time points [11]. However, these findings are exploratory and require further investigation in the in vivo and in vitro mechanistic studies.

\section{Conclusions}

Left ventricular remodeling is identified as a central pathophysiologic mechanism involved in the development of HF. Finding reliable biomarkers for left ventricular remodeling may facilitate the risk stratification and prognosis assessment of patients with HF. The results of the present study suggested that the expression levels of plasma miR-20a-5p, miR-340-5p, and let-7i-5p were associated with the development of left ventricular remodeling. The expression levels of plasma miR-20a-5p presented significant correlations with left ventricular dilatation in rats and patients with postinfarction HF. Therefore, miR-20a-5p may serve as a novel potential biomarker for cardiac remodeling during the development of postinfarction HF.

\section{Data Availability}

The database used to support the findings of this study is available from the corresponding author upon request.

\section{Conflicts of Interest}

The authors declare that there is no conflict of interest regarding the publication of this paper.

\section{Acknowledgments}

This work was supported by the National Natural Science Foundation of China (grant number 81570360).

\section{Supplementary Materials}

Supplementary Figure 1: expression levels of miR-20a-5p, miR-340-5p, and let-7i-5p in the liver, spleen, lung, kidney, and thymus tissues in the 8-week post-MI group and the sham group. Supplementary Table 1: the KEGG annotation results of the 19 common target genes. (Supplementary Materials)

\section{References}

[1] P. Ponikowski, A. A. Voors, S. D. Anker et al., "2016 ESC guidelines for the diagnosis and treatment of acute and chronic heart failure: the task force for the diagnosis and treatment of acute and chronic heart failure of the European Society of Cardiology (ESC) developed with the special contribution of the Heart Failure Association (HFA) of the ESC," European Heart Journal, vol. 37, no. 27, pp. 2129-2200, 2016.

[2] M. A. Konstam, D. G. Kramer, A. R. Patel, M. S. Maron, and J. E. Udelson, "Left ventricular remodeling in heart failure: current concepts in clinical significance and assessment," JACC: Cardiovascular Imaging, vol. 4, no. 1, pp. 98-108, 2011.

[3] G. Heusch, P. Libby, B. Gersh et al., "Cardiovascular remodelling in coronary artery disease and heart failure," Lancet, vol. 383, no. 9932, pp. 1933-1943, 2014.

[4] M. A. Pfeffer and E. Braunwald, "Ventricular remodeling after myocardial infarction. Experimental observations and clinical implications," Circulation, vol. 81, no. 4, pp. 11611172, 1990.

[5] E. A. van Vliet, N. Puhakka, J. D. Mills et al., "Standardization procedure for plasma biomarker analysis in rat models of epileptogenesis: focus on circulating microRNAs," Epilepsia, vol. 58, no. 12, pp. 2013-2024, 2017.

[6] T. Thum and G. Condorelli, "Long noncoding RNAs and microRNAs in cardiovascular pathophysiology," Circulation Research, vol. 116, no. 4, pp. 751-762, 2015.

[7] A. Castaldi, T. Zaglia, V. Di Mauro et al., "MicroRNA-133 modulates the $\beta 1$-adrenergic receptor transduction cascade," Circulation Research, vol. 115, no. 2, pp. 273-283, 2014.

[8] I. Karakikes, A. H. Chaanine, S. Kang et al., "Therapeutic cardiac-targeted delivery of miR-1 reverses pressure overload-induced cardiac hypertrophy and attenuates pathological remodeling," Journal of the American Heart Association, vol. 2, no. 2, article e000078, 2013.

[9] R. L. Montgomery, T. G. Hullinger, H. M. Semus et al., “Therapeutic inhibition of miR-208a improves cardiac function and survival during heart failure," Circulation, vol. 124, no. 14, pp. 1537-1547, 2011.

[10] E. E. Creemers, A. J. Tijsen, and Y. M. Pinto, "Circulating microRNAs: novel biomarkers and extracellular communicators in cardiovascular disease?," Circulation Research, vol. 110, no. 3, pp. 483-495, 2012.

[11] K. M. Danielson, R. Shah, A. Yeri et al., "Plasma circulating extracellular RNAs in left ventricular remodeling postmyocardial infarction," eBioMedicine, vol. 32, pp. 172-181, 2018.

[12] R. Shah, O. Ziegler, A. Yeri et al., "MicroRNAs associated with reverse left ventricular remodeling in humans identify pathways of heart failure progression," Circulation. Heart Failure, vol. 11, no. 2, article e004278, 2018.

[13] P. Lv, M. Zhou, J. He et al., "Circulating miR-208b and miR34a are associated with left ventricular remodeling after acute myocardial infarction," International Journal of Molecular Sciences, vol. 15, no. 4, pp. 5774-5788, 2014.

[14] D. de Gonzalo-Calvo, G. Cediel, C. Bar et al., "Circulating miR-1254 predicts ventricular remodeling in patients with ST-segment-elevation myocardial infarction: a cardiovascular magnetic resonance study," Scientific Reports, vol. 8, no. 1, p. 15115, 2018.

[15] C. Bauters, R. Kumarswamy, A. Holzmann et al., "Circulating miR-133a and miR-423-5p fail as biomarkers for left 
ventricular remodeling after myocardial infarction," International Journal of Cardiology, vol. 168, no. 3, pp. 1837-1840, 2013.

[16] S. C. Latet, P. L. Van Herck, M. J. Claeys et al., "Failed downregulation of circulating microRNA-155 in the early phase after ST elevation myocardial infarction is associated with adverse left ventricular remodeling," Cardiology, vol. 138, no. 2, pp. 91-96, 2017.

[17] U. Grabmaier, S. Clauss, L. Gross et al., "Diagnostic and prognostic value of miR-1 and miR-29b on adverse ventricular remodeling after acute myocardial infarction - the SITAGRAMI-miR analysis," International Journal of Cardiology, vol. 244, pp. 30-36, 2017.

[18] Y. Devaux, M. Vausort, G. P. McCann et al., "MicroRNA-150: a novel marker of left ventricular remodeling after acute myocardial infarction," Circulation. Cardiovascular Genetics, vol. 6, no. 3, pp. 290-298, 2013.

[19] S. Goldman and T. E. Raya, "Rat infarct model of myocardial infarction and heart failure," Journal of Cardiac Failure, vol. 1, no. 2, pp. 169-177, 1995.

[20] K. Thygesen, J. S. Alpert, A. S. Jaffe et al., "Fourth universal definition of myocardial infarction (2018)," Journal of the American College of Cardiology, vol. 72, no. 18, pp. 2231-2264, 2018.

[21] M. Writing Committee, C. W. Yancy, M. Jessup et al., "2013 ACCF/AHA guideline for the management of heart failure: a report of the American College of Cardiology Foundation/American Heart Association task force on practice guidelines," Circulation, vol. 128, no. 16, pp. e240-e327, 2013.

[22] Authors/Task Force members, S. Windecker, P. Kolh et al., "2014 ESC/EACTS guidelines on myocardial revascularization," European Heart Journal, vol. 35, no. 37, pp. 25412619, 2014.

[23] J. C. Huang, T. Babak, T. W. Corson et al., "Using expression profiling data to identify human microRNA targets," Nature Methods, vol. 4, no. 12, pp. 1045-1049, 2007.

[24] Y. P. Wang and K. B. Li, "Correlation of expression profiles between microRNAs and mRNA targets using NCI-60 data," BMC Genomics, vol. 10, p. 218, 2009.

[25] M. D. Dennis, N. K. McGhee, L. S. Jefferson, and S. R. Kimball, "Regulated in DNA damage and development 1 (REDD1) promotes cell survival during serum deprivation by sustaining repression of signaling through the mechanistic target of rapamycin in complex 1 (mTORC1)," Cellular Signalling, vol. 25, no. 12, pp. 2709-2716, 2013.

[26] L. C. Caetano, M. L. Bonfleur, R. A. Ribeiro et al., "Taurine supplementation regulates $\mathrm{I} \kappa-\mathrm{B} \alpha$ protein expression in adipose tissue and serum IL- 4 and TNF- $\alpha$ concentrations in MSG obesity," European Journal of Nutrition, vol. 56, no. 2, pp. 705-713, 2017.

[27] E. M. Seymour, M. R. Bennink, S. W. Watts, and S. F. Bolling, "Whole grape intake impacts cardiac peroxisome proliferatoractivated receptor and nuclear factor kappaB activity and cytokine expression in rats with diastolic dysfunction," Hypertension, vol. 55, no. 5, pp. 1179-1185, 2010.

[28] G. Sun, R. Xue, F. Yao et al., "The critical role of sestrin 1 in regulating the proliferation of cardiac fibroblasts," Archives of Biochemistry and Biophysics, vol. 542, pp. 1-6, 2014.

[29] J. H. Lee, A. V. Budanov, E. J. Park et al., "Sestrin as a feedback inhibitor of TOR that prevents age-related pathologies," Science, vol. 327, no. 5970, pp. 1223-1228, 2010.

[30] B. Swynghedauw, "Molecular mechanisms of myocardial remodeling,” Physiological Reviews, vol. 79, no. 1, pp. 215-262, 1999.
[31] E. Braunwald and M. A. Pfeffer, "Ventricular enlargement and remodeling following acute myocardial infarction: mechanisms and management," The American Journal of Cardiology, vol. 68, no. 14, pp. 1D-6D, 1991.

[32] E. M. Small and E. N. Olson, "Pervasive roles of microRNAs in cardiovascular biology," Nature, vol. 469, no. 7330 , pp. 336342, 2011.

[33] S. P. Romaine, M. Tomaszewski, G. Condorelli, and N. J. Samani, "MicroRNAs in cardiovascular disease: an introduction for clinicians," Heart, vol. 101, no. 12, pp. 921-928, 2015.

[34] K. W. Witwer, "Circulating microRNA biomarker studies: pitfalls and potential solutions," Clinical Chemistry, vol. 61, no. 1, pp. 56-63, 2015.

[35] R. V. Shah, J. Rong, M. G. Larson et al., "Associations of circulating extracellular RNAs with myocardial remodeling and heart failure," JAMA Cardiology, vol. 3, no. 9, pp. 871-876, 2018.

[36] J. A. Park and C. H. Lee, "Time-course change of Redd1 expressions in the hippocampal CA1 region following chronic cerebral hypoperfusion," Cellular and Molecular Neurobiology, vol. 37, no. 3, pp. 563-569, 2017.

[37] C. Liu, R. Xue, D. Wu et al., "REDD1 attenuates cardiac hypertrophy via enhancing autophagy," Biochemical and Biophysical Research Communications, vol. 454, no. 1, pp. 215220, 2014.

[38] M. Laplante and D. M. Sabatini, "mTOR signaling in growth control and disease," Cell, vol. 149, no. 2, pp. 274-293, 2012.

[39] S. Sciarretta, M. Forte, G. Frati, and J. Sadoshima, "New insights into the role of mTOR signaling in the cardiovascular system," Circulation Research, vol. 122, no. 3, pp. 489-505, 2018.

[40] A. V. Budanov and M. Karin, "p53 target genes sestrin1 and sestrin2 connect genotoxic stress and mTOR signaling," Cell, vol. 134, no. 3, pp. 451-460, 2008.

[41] J. W. Gordon, J. A. Shaw, and L. A. Kirshenbaum, "Multiple facets of NF- $\kappa \mathrm{B}$ in the heart: to be or not to NF- $\kappa \mathrm{B}$," Circulation Research, vol. 108, no. 9, pp. 1122-1132, 2011.

[42] W. Luo, G. Li, Z. Yi, Q. Nie, and X. Zhang, "E2F1-miR-20a$5 \mathrm{p} / 20 \mathrm{~b}-5 \mathrm{p}$ auto-regulatory feedback loop involved in myoblast proliferation and differentiation," Scientific Reports, vol. 6, p. 27904, 2016.

[43] Y. Hu, G. Jin, B. Li et al., "Suppression of miRNA let-7i-5p promotes cardiomyocyte proliferation and repairs heart function post injury by targetting CCND2 and E2F2," Clinical Science, vol. 133, no. 3, pp. 425-441, 2019.

[44] J. Zhang, J. Ma, K. Long et al., "Overexpression of exosomal cardioprotective miRNAs mitigates hypoxia-induced H9c2 cells apoptosis," International Journal of Molecular Sciences, vol. 18, no. 4, 2017.

[45] E. L. Vegter, E. S. Ovchinnikova, D. J. van Veldhuisen et al., "Low circulating microRNA levels in heart failure patients are associated with atherosclerotic disease and cardiovascularrelated rehospitalizations," Clinical Research in Cardiology, vol. 106, no. 8, pp. 598-609, 2017.

[46] N. Bruno, J. M. ter Maaten, E. S. Ovchinnikova et al., "MicroRNAs relate to early worsening of renal function in patients with acute heart failure," International Journal of Cardiology, vol. 203, pp. 564-569, 2016.

[47] C. Voellenkle, J. van Rooij, C. Cappuzzello et al., "MicroRNA signatures in peripheral blood mononuclear cells of chronic heart failure patients," Physiological Genomics, vol. 42, no. 3, pp. $420-426,2010$. 


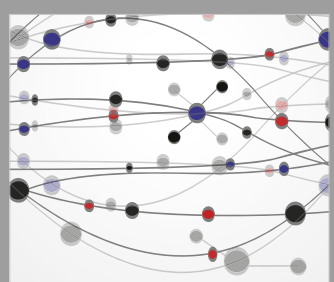

The Scientific World Journal
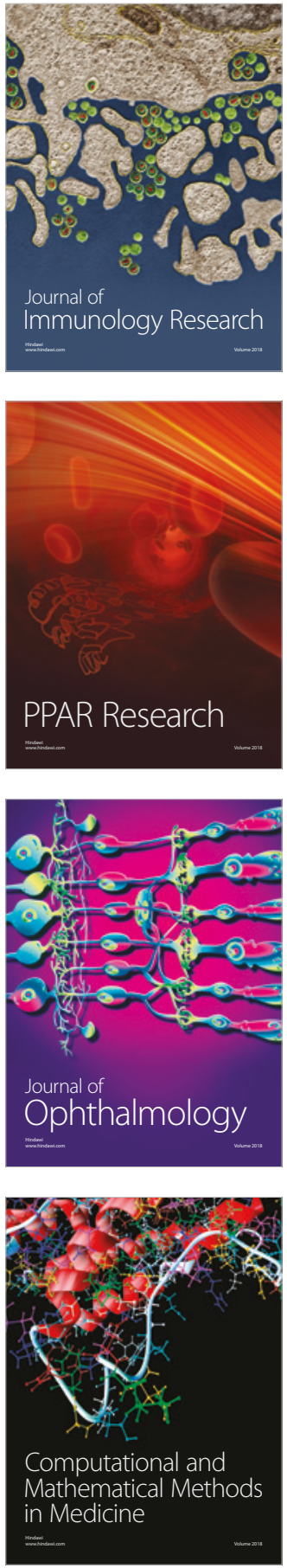

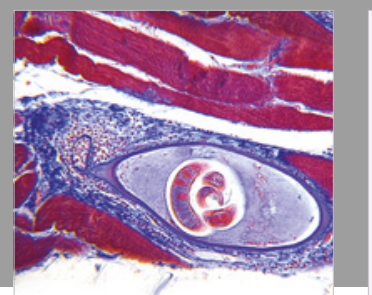

Gastroenterology Research and Practice

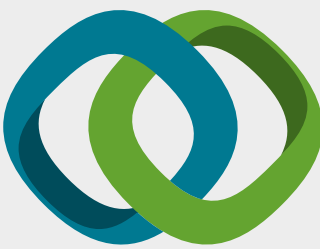

\section{Hindawi}

Submit your manuscripts at

www.hindawi.com
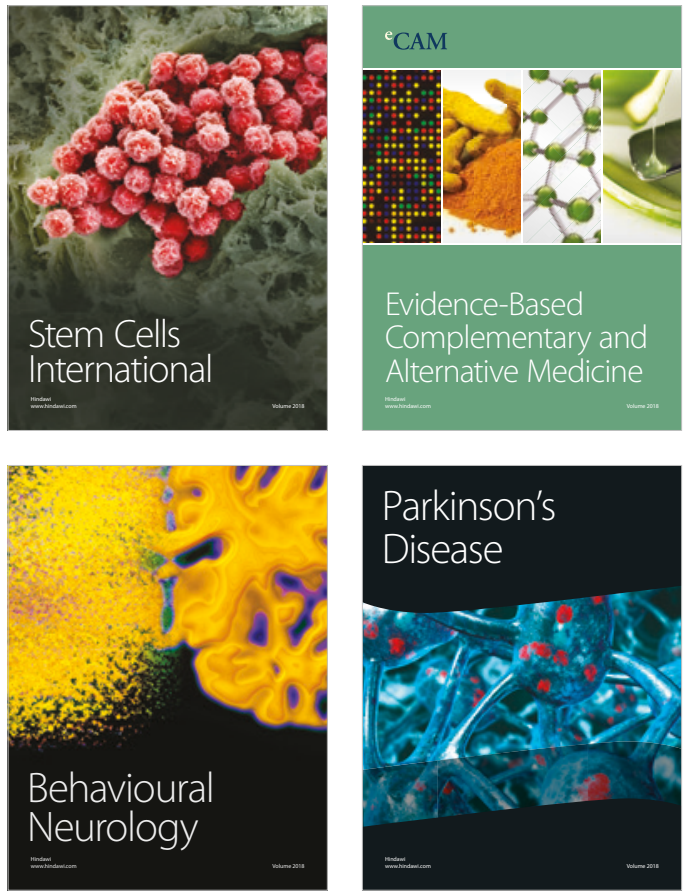

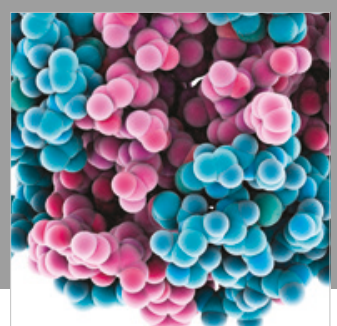

ournal of

Diabetes Research

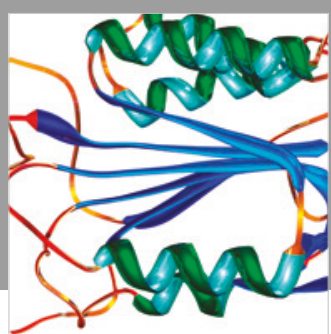

Disease Markers
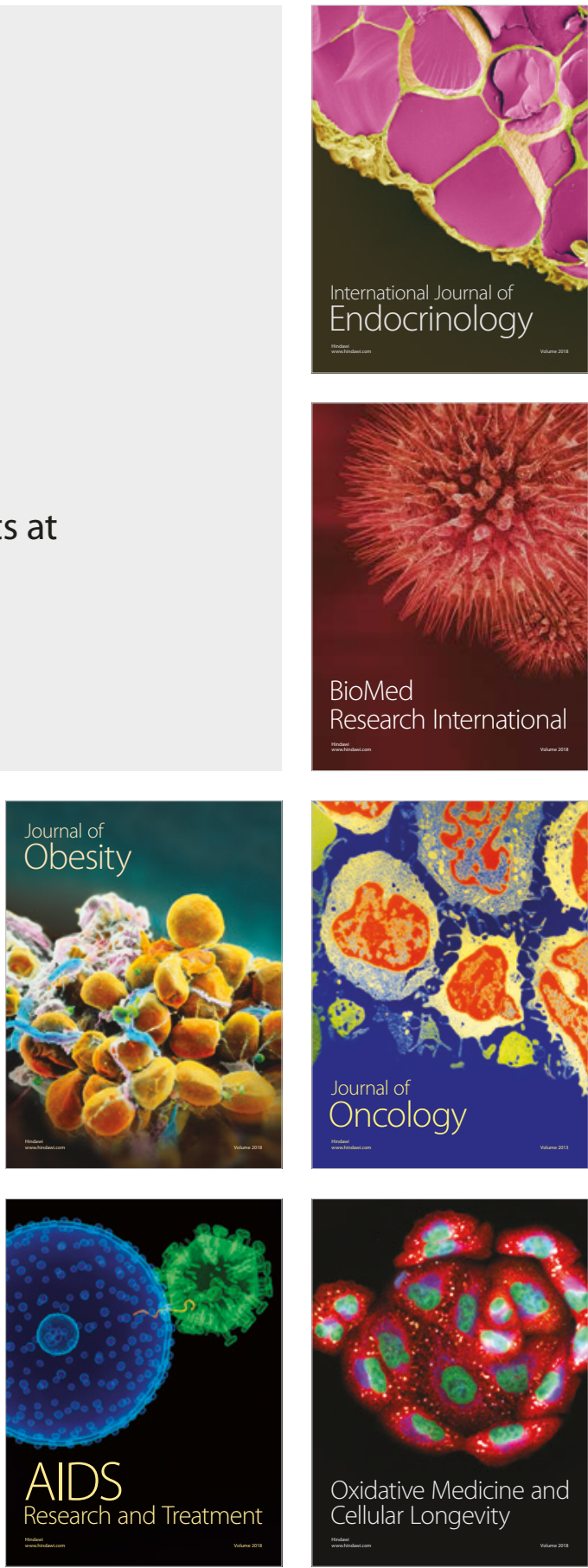